\title{
Communication training and its effects on carer and care- receiver outcomes in dementia settings: A systematic review
}

\section{Abstract}

Aims: To review communication interventions that aim to improve regular care interactions between people with dementia and their carers in various settings; and to examine the impact of such interventions on both carer and care-receiver outcomes.

Background: Effective communication is imperative to ensure quality of care for people living with dementia. Due to neurodegenerative changes, people with dementia encounter ongoing and progressive difficulties in both understanding and expressing themselves. This in turn creates challenges for carers, which highlights the need for equipping them with necessary communication skills to respond to the specific communication needs of people with dementia.

Design: Systematic review and meta-analysis

Method: Medline, Embase, CINAHL, ProQuest, and PsycINFO databases were searched for eligible interventions with any date of the publication. Hand searching was also conducted through reviewing the reference lists of relevant articles. The screening and selection of studies were based on the inclusion/exclusion criteria for eligibility and the methodological quality assessment checklist. Random-effects meta-analyses were conducted on comparable quantitative data. The review is reported following the PRISMA reporting guidelines.

Results: 17 studies were included in the final review, including 12 randomised controlled trials (RCTs), three non-randomised controlled trials (NRCTs), and two controlled beforeafter interventions (CBAs). The intervention designs, settings, and outcome measures were varied. The findings suggest that the communication training had a positive impact on both carer and care-receiver outcomes, albeit to different degrees. The intervention effects were found to be strongest on carer communication skills and knowledge.

Conclusion: There is solid evidence for the positive impact of communication training on the skills and knowledge of carers. More research is needed regarding the effects of such educational interventions on carer physio-psychological outcomes and care-receiver neuropsychiatric symptoms. It is important to establish best practices in training design, develop validated outcome measures, and adopt consistent reporting approaches.

Relevance to Clinical Practice: The increasing global prevalence of people with dementia manifests across clinical and community contexts. The profound impact of dementia on communication and associated care raises the imperative for enhanced health worker and carer communication skills to meet the needs of this particular client group. The findings of this review indicate that educational interventions incorporating face-to-face and diverse instructional delivery methods in dementia communication showed positive outcomes for communication skills in all carer groups and warrant inclusion as strategies in dementia training.

Key words: Dementia care, Communication training, Systematic review

What does this paper contribute to the wider global clinical community?

- Dementia is a major and growing health issue of global concern and relevance 
- Skilled health worker communication is fundamental to clinical outcomes for people living with dementia

- This review identifies that face-to-face and diverse instructional delivery in dementia education and training are strategies that increase clinical communication skills and should be considered when developing such interventions for all health workers

\section{Introduction}

Communication is fundamental to human interaction. Communication is built on both vocal and visible cues that are simultaneously used to create an interactive event (Buck \& VanLear, 2002). As people communicate verbally, body posture, hand movement, facial expression, touch, and eye contact are also used as part of this social interaction to convey particular messages between two or more parties (Jones \& LeBaron, 2002; Machiels, Metzelthin, Hamers, \& Zwakhalen, 2017). Within healthcare, much like other aspects of life, effective communication is a fundamental element to ensure the provision of quality care. In contrast, communication that is fragmented or interrupted may impact negatively on patient functioning, comfort, safety and the care provision (Machiels et al., 2017).

Owing to neurodegenerative changes, people with dementia are often faced with difficulties in word finding, sound production, reduced fluency, or comprehension of language, depending on the progression of the condition (Allan \& Killick, 2014). Their capacity to communicate can be further undermined by depression, a loss of confidence, anxiety, and other psychological factors (Downs \& Bowers, 2014). However, people living with dementia may retain the ability to communicate using short and simple word structures and more direct non-verbal cues (Hubbars, Cook, Tester, \& Downs, 2002; Machiels et al., 2017). These patterns of change vary between individuals; however, communication difficulties tend to be progressive (Bryan \& Maxim, 2006).

There is increasing evidence that communication between people with dementia and their carers is problematic, which has substantial consequences for the quality of care provided, quality of life, wellbeing and the behaviours of individuals with dementia (Alnes, Kirkevold, \& Skovdahl, 2011; Eggenberger, Heimerl, \& Bennett, 2013). Observed care interactions are often infrequent, brief, impoverished and task-oriented, partly due to the generally perceived inability to communicate of those with dementia as well as organizational factors (Ward, Vass, Aggarwal, Garfield, \& Cybyk, 2008). This contributes to a situation of unmet need and a strain on the carer and care-receiver relationship, which possibly contributes to behavioural and psychological symptoms among people with dementia (Downs \& Collins, 2015). On the carer's part, higher levels of stress and burnout may also be linked to a lack of skill in addressing the communication needs of individuals with dementia in daily care (Beer, Hutchinson, \& Skala-Cordes, 2012; Broughton et al., 2011).

Supporting the growing number of people with dementia worldwide (Prince, Comas-Herrera, Knapp, Guerchet, \& Karagiannidou, 2016) includes the provision of adequate and effective training/education that encompasses specific communication skills and strategies to facilitate improved communication in both professional and non-professional dementia care settings (Eggenberger et al., 2013). Findings from the literature suggest that increasing communication competence for carers contributes positively to the well-being of people with dementia as well as their carers. For example, in their interventions aimed to enhance social communicative competencies of family and professional carers, Krause and colleagues (2009) found an increase in the well-being and communication ability of people with dementia and a reduction in carer burden. Similarly, Haberstroh, Neumeyer, Schmitz, and 
Pantel (2009) reported increased quality of life of people with dementia and decreased carer stress, as a result of communication training for residential care staff. With a focus on the carer, McGuire (1995) and Auer, Donabauer, Zehetner, and Span (2007) found evidence for communication training resulting in reduced carer stress, burden and depressive symptoms. These positive outcomes could be largely explained by the widely-noted better communicative behaviour of carers after training (e.g. Levy-Storms, Harris, \& Chen, 2016; Roque, Ortiz, Araujo Mda, \& Bertolucci, 2009; Williams, 2006) and improved carer - care receiver relationship and communication during care routines (e.g. Allen-Burge, Burgio, Bourgeois, \& Nunnikhoven, 2001; Berwig, Dinand, \& Halek, 2016).

Previous systematic reviews have also found evidence, albeit inconsistent, for the positive impact of different communication programs on both carers and people with dementia. Vasse, Vernooij-Dassen, Spijker, Rikkert, and Koopmans (2010) conducted a systematic review of 10 set-time interventions (e.g. walk-and-talk, group validation, cognitive stimulation or life-review) and nine daily-care interventions solely or partially aimed at improving care staff's communication techniques (e.g. non-verbal sensitivity, behaviour management, emotion-oriented care, abilities-focused and needs-based engagement, or general communication enhancing strategies). They found positive effects on communication especially in interactive behaviours and positive affect in most daily-care interventions, and noted the inclusion of interactive sessions, self-study, and individual attention among the successful programs. However, they found only a marginal impact on neuropsychiatric symptoms of people with dementia in residential care settings. Similarly, in their systematic review of 12 communication training interventions (e.g. verbal, non-verbal and emotional skills, attitudes, behavioural management, usage of tools, or theoretical knowledge) for both professional and non-professional carers of people with dementia, Eggenberger et al. (2013) reported evidence for better communication competence among carers, improved interactions, and enhanced quality of life for people with dementia as a result of such training. The authors highlighted that the more effective and sustainable interventions incorporated additional supportive features (e.g. feedback or self-evaluation) and follow-up measures. A more recent systematic review of six interventions focused on daily nursing care communication between nursing staff and people with dementia by Machiels et al. (2017) also reported positive effects on verbal and non-verbal communication, with one or more communication outcomes demonstrating statistically significant results in most studies. The authors, however, emphasised the difficulty in drawing conclusions about training effectiveness due to the small number of reviewed interventions, their variation in content and outcome measures, as well as methodological shortcomings.

While the previous reviews have provided insights into the evaluation of communication training for carers of people with dementia, they tended to focus only on residential aged care settings (Machiels et al., 2017; Vasse et al., 2010) or only on carer communication outcomes (Machiels et al., 2017). In addition, none of the three reviews included studies outside of Europe and North America; nor did they examine the training impact on carer psychophysiological outcomes.

This systematic review and meta-analysis aims to evaluate communication training interventions relevant to regular care interactions between carers and people with dementia in both residential and home settings across different countries and regions; and examine their impact on carer communication, carer psycho-physiological states, as well as neuropsychiatric symptoms of people with dementia. 


\section{Methods}

The current systematic review and meta-analysis was guided by the Cochrane Handbook for Systematic Reviews of Interventions to identify, appraise, and synthesise research-based evidence (Higgins \& Green, 2008). The objectives, analysis methods and inclusion criteria were developed in advance and documented in a protocol. The PRISMA reporting guidelines (Supplementary Table 1 in the Appendices) were consulted and adhered to with a view to ensuring accurate and complete reporting of the systematic review findings.

\section{Search strategy}

Medline, Embase, CINAHL, ProQuest, and PsycINFO databases were accessed for communication training intervention studies with any date of the publication. The databases were accessed by title, keyword, or abstract and then full-text. Search terms included: 'Dementia' OR 'Alzheimer' OR 'Alzheimer's disease' OR 'Vascular dementia' OR 'Lewy Body' OR 'Lewy Body disease' OR 'Frontotemporal dementia' AND 'Communication' OR 'communicating' OR 'communicate' OR 'interaction' OR 'interact' OR 'interacting' OR 'conversation' OR 'talk' OR 'talking' OR 'verbal' OR 'non-verbal' AND 'training' OR 'intervention' OR 'trial' OR 'experiment' OR 'program' AND 'effectiveness' OR 'effect' OR 'impact' OR 'result' OR 'outcome'.

This strategy was used to search title and abstract in all databases and was adapted to the specific requirements of each database. Additional searches were conducted using hand searching or reviewing reference lists. Lastly, correspondence with key authors was undertaken to seek clarification and to ensure eligibility criteria was met.

\section{Inclusion and exclusion criteria}

The reviewed studies were those designed around the implementation and evaluation of an intervention to improve verbal and/or nonverbal communication in regular day-to-day interactions between people with dementia and their carers. In particular, the participants in the interventions were carers in both residential settings and home care settings. The participating care-receivers were people with a diagnosis of dementia at different stages (mild, moderate and severe), of any age group and receiving care in residential or home environments. The reviewed studies were randomised controlled trials (RCTs), nonrandomised controlled trials (NRCTs), or controlled before and after interventions (CBAs), all of which involved a comparison of observations between an intervention group and a control group.

Multi-component interventions that targeted regular care communication skills for carers of people with dementia as part of their training program were included; however, studies were excluded if they focused only on therapies to aid memory, sensory perception, or physical/mental functions, such as reminiscence therapy, music therapy, doll therapy, play therapy, gardening, art-based programs, aromatherapy, bright light therapy, and Snoezelen. Although these therapies might involve communication to different extents, they principally facilitate fix-timed interactions, rather than regular care interactions. In addition, if the interventions covered only theoretical knowledge of dementia, they were excluded. Full-text articles published in languages other than English were not reviewed because of difficulties in ensuring translation qualities. 


\section{Study screening}

The studies that were retrieved from all databases were exported to EndNote (version X7). The screening and selection of studies were mainly performed by two reviewers ( $\mathrm{HN}$ and DT) after duplicates were removed. In the first round, the two reviewers independently screened all studies based on titles, keywords and abstracts to exclude obviously irrelevant records. This resulted in the identification of potentially relevant studies for full text review. In the second round, the full texts were again assessed independently by the two reviewers against the inclusion and exclusion criteria for eligibility. Each study was classified as 'include', 'exclude' or 'not sure' in the review form. Any discrepancies between the two reviewers were resolved by discussion until a consensus was reached and/or through discussion with a third reviewer (HP). A citation and reference check was also performed on these full-text articles by the third reviewer (HP), and the results were then discussed among the three reviewers to ensure all relevant articles were included. At each round, each article and the reason for inclusion and exclusion were documented.

\section{Methodological quality assessment procedure}

The methodological quality of the reviewed studies was assessed independently by two reviewers, using a checklist with a number of criteria in alignment with the Cochrane guidelines (Higgins \& Green, 2008). Specifically, the quality assessment criteria addressed the five common types of bias in research studies, namely selection bias, performance bias, attrition bias, detection bias, and reporting bias. These criteria were rated as 'criterion met' $(+)$, 'criterion not met' (-), 'unknown if the criterion is met or not met' (u), and 'not applicable' (n/a). Any differences in the quality assessment results between the two reviewers were discussed, and the third reviewer was also consulted to reach consensus.

\section{Data extraction and analysis}

A data extraction form was developed to meet the needs of the review. Data from all eligible studies were extracted on key aspects such as research design and participants, study settings, intervention and materials, training duration and formats, how outcomes were measured, and the intervention outcomes. The data extraction form was completed by one researcher $(\mathrm{HN})$ and discussed with the second and third researchers (DT and HP) until a consensus regarding all details was reached. If data were missing or lacking, the researchers contacted the authors of the specific articles to ask for more detailed information regarding the methods of the intervention used and results.

The studies with comparable quantitative data were pooled in five meta-analyses to measure the intervention effects on carer communication outcomes, carer psycho-physiological outcomes, and neuropsychiatric symptoms of people with dementia. The pooled data were analysed in Stata 12.1. All two-tailed p-values less than 0.05 were considered as statistically significant.

To prepare for the meta-analyses, data from eligible studies were extracted with mean values of outcome and standard deviation (SD) separately for the intervention and control groups at baseline, post-training, and/or follow-up (FUP). We then, where possible, calculated the mean difference (MD) and SD for the change in mean scores between pre- and posttraining/FUP. Because outcome measures were based on different scales and instruments, calculations of standardised mean difference (SMD) were performed to estimate the effect of training based on the score change between pre- and post-intervention/FUP in each group. Finally, for each outcome, study-specific SMD estimates were combined using randomeffects meta-analysis because of inconsistent designs, settings and outcome measures 
between the studies from different populations. Heterogeneity was evaluated using Q, $\tau^{2}$, and $\mathrm{I}^{2}$ statistics. Meta-regression was used to account for sources of heterogeneity among studylevel characteristics, including study design (RCT, NRCT, CBA), setting (residential and home-based settings), outcome measures (questionnaire, knowledge test, and observation checklist), and region (Europe, North America, and Australasia). Publication bias was assessed using the visual funnel plot and Begg's test (Begg \& Mazumdar, 1994; Egger, Smith, Schneider, \& Minder, 1997). An asymmetric plot or a p-value being $\leq 0.10$ (Egger's test) would suggest a possible publication bias, which would then necessitate an updated estimation of the pooled effect size using Duval and Tweedie's trim and fill method to see whether the hypothetical 'missing' studies affected our results (Duval \& Tweedie, 2000).

\section{Findings}

The extensive search for relevant studies from multiple databases resulted in a total of 2,817 records. 46 additional records were identified via other sources. After 1,128 duplicates were removed, there were 1,735 potentially relevant records. The subsequent title and abstract screening led to the exclusion of 1,673 records, and five records were also excluded because full texts were not available. A total of 57 full-text articles were assessed for eligibility against the inclusion and exclusion criteria, resulting in 40 studies being removed from the review. There were several reasons for exclusion, for example study design (such as the absence of an intervention or a control group), systematic review papers, a lack of focus on regular care communication between people with dementia and their carers, or the absence of full-texts in English. Finally, 17 studies were included in the systematic review and metaanalysis where the necessary data was available. Figure 1 provides an overview of the literature search process and results.

\section{Methodological quality assessment results}

The methodological quality of the reviewed studies varied, with the RCTs tending to meet more quality criteria than NRCTs and CBAs. The criteria were marked as 'criterion met', 'criterion not met', 'unknown', or 'not applicable' based on the information provided in the paper. It was worth noting that although 12 studies were identified as RCTs, only three articles included adequate details of how randomization and concealment were performed (Barnes, 2016; Done \& Thomas, 2001; Kuske et al., 2009). Blinding of outcome assessors were reported in several studies, whereas blinding of participants was not mentioned by most studies. However, due to the nature of the intervention, where carer participants were invited to provide written consent, it is arguably hard to fulfil this criterion (Eggenberger et al., 2013). The two criteria that were satisfied by the majority of the reviewed studies are the description of participant inclusion/exclusion criteria and non-selective reporting of outcomes. On the other hand, only one study (Barnes, 2016) followed the intention-to-treat principle. The review authors' judgements about risks of bias of each study are presented in Supplemental Table 2 in the Appendices.

\section{Publication bias assessment}

Our visual inspection of the funnel plots (Supplemental Figures 1-5) and results from Begg's tests (Supplemental Table 3) showed very little evidence of publication bias in this review. The only possibility of publication bias was observed among the studies reporting changes of carer psycho-physiological states between baseline and follow-up outcomes (Begg's test; $p=0.007$; Supplemental Figure 4). However, our sensitivity analysis using the 'trim and fill' 
method revealed that the updated pooled estimate remained unchanged (pooled SMD 0.30, $95 \%$ CI $0.01,0.59)$, suggesting our results were robust.

\section{Description of the reviewed studies}

The reviewed studies were conducted between 1998 and 2016 in North America (e.g. the USA, Canada), Europe (e.g. the Netherlands, Germany, the UK, Norway), and Australasia (e.g. Australia), and various residential settings (11 studies) and home-based settings (six studies). Regarding research design, twelve studies were RCTs; three studies were NRCTs, and two were CBAs. While all studies involved a control group to allow for comparison with the intervention group, the nature of the comparison treatment differed. The control group of carers in 12 studies received no intervention whereas those in five studies received placebo treatments, such as training on a different topic (Barnes, 2016; Kuske et al., 2009; Magai et al., 2002) or provision of a booklet or information flier (Done \& Thomas, 2001; Kouri et al., 2011).

There were a total of 794 carers participating in the 17 reviewed studies. Among them, 527 were residential carers, who had direct contact with people with dementia and who were working as either a registered nurse, registered practical nurse, enrolled nurse, endorsed enrolled nurse, licensed practical nurse, certified nurse/nursing assistant, nursing assistant, nursing aide, or recreational/activities officer/coordinator. These residential carers were reported to come from different residential settings, including community aged care services, nursing homes, residential aged care facilities, dementia-specific care units, and cognitive support units. The remaining 267 carers were home-based/family carers, who were spouses, partners, children, or friends of people with dementia and who identified themselves as the principal/primary co-habiting carer or who had daily/regular contact with the person living with dementia. Each study involved from 12 (Dampney-Jay, 2015) to 96 (Kuske et al., 2009) residential carers, and from 29 (Liddle et al., 2012) to 54 (Ripich et al., 1999) homebased/family carers.

The number of people with dementia participating in the reviewed studies also varied. In residential settings, nine studies reported the involvement of 631 people with dementia in total, ranging from 10 (Alnes et al., 2011) to 210 (Kuske et al., 2009) people in each study, with missing information in two studies. In home-based settings, one study reported the participation of 29 people with dementia (Liddle et al., 2012) while five studies did not report on this information.

As indicated by the Mini-Mental State Examination or Dementia Severity Rating Scale scores, the care-receivers' severity of dementia differed within and across the studies. Eight studies involved people with dementia spanning a range of severity, namely mild, moderate, and severe levels (Burgio et al., 2001; Burgio et al., 2002; Kuske et al., 2009; Liddle et al., 2012; McCallion et al., 1999; Ripich et al., 1998; Ripich et al., 1999; Sprangers et al., 2015). Four studies engaged people with higher severity, i.e. moderate and/or severe dementia (Alnes et al., 2011; Dampney, 2015; Magai et al., 2002; Wells et al., 2000), while two studies involved participants with mild dementia only (Barnes, 2016; Kouri et al., 2011). Dementia severity was not reported in three studies, possibly because people with dementia were not considered their target participants. Table 1 provides an overview of the studies in the review.

\section{Intervention contents}

The reviewed studies reported on a wide range of communication training programs that were aimed at improving the regular day-to-day interactions between carers and people with dementia. Many of these programs were newly developed by the author researchers, for 
example, the Talking Sense by Barnes (2016), the Nursing Assistant Communication Skills Program by McCallion et al. (1999), the FOCUS Program by Ripich et al. (1998), the Abilities-focused Program of Care by Wells (2000), and other communication skills training by Spranger et al. (2015), Burgio et al. (2001), or Done and Thomas (2001). Some communication training programs were adopted or adapted from those developed by previous researchers, such as The Intensive Interaction Training (Dampney-Jay, 2015), the RECAPS and MESSAGE program (Broughton et al., 2011; Conway \& Chenery, 2016; Liddle et al., 2012), the Eight-Function Supporting Elements Program (Alnes et al., 2011), or the Psychoeducational Program about Communication (Kouri et al., 2011).

The core focus of all interventions was on the use of verbal skills (e.g. language choice, speech rate, tone, volume) and/or non-verbal skills (e.g. eye contact, posture, gesture, smile) to perform various speech functions in regular interactions with people with dementia. Those functions included directing and maintaining attention, facilitating understanding, giving instructions, making announcements, providing support and encouragement, or showing interest. In some multi-component interventions, additional communication aspects were also covered, including behavioural management skills (Alnes et al., 2011; Barnes, 2016); creating favourable environment for communication (Broughton et al., 2011; Burgio et al., 2002; Liddle et al., 2012); use of communication support tools such as memory books or visual aids (Broughton et al., 2011; Conway \& Chenery, 2016; Liddle et al., 2012; McCallion et al., 1999); and theoretical knowledge about dementia and its impact on communication (Barnes, 2016; Kuske et al., 2009; McCallion et al., 1999).

\section{Intervention methods and materials}

All of the communication training programs under review were conducted face-to-face. The training facilitators were the author researchers and/or other specialists, who were either a psychologist (Burgio et al., 2001; Broughton et al., 2011; Conway \& Chenery, 2016; Dampney, 2015; Liddle et al., 2012; Kuske et al., 2009; Magai et al., 2002), geropsychologist (Burgio et al., 2002); speech/language pathologist (Broughton et al., 2011; Conway \& Chenery, 2016; Done \& Thomas, 2001; Liddle et al., 2012; Ripich et al., 1998; Ripich et al., 1999); trained nurse practitioner (Kouri et al., 2011); or Master's level social worker (McCallion et al., 1999). The training facilitator was not explicitly identified in one study (Sprangers et al., 2015).

In residential care settings, all interventions were organised in groups, mostly with videobased presentations and/or demonstrations followed by group discussions and activities such as role-plays, games or exercises. Some interventions incorporated hands-on training with counselling and feedback on real interactions between the participating carers and people with dementia (Alnes et al., 2011; Burgio et al., 2001; Burgio et al., 2002; Conway \& Chenery, 2016; Dampney, 2015; Sprangers et al., 2015). Additional instructional materials were used, such as booklets, posters (Broughton et al., 2011), manuals (Wells et al., 2000), workbooks (Burgio et al., 2002; McCallion et al., 1999) and handouts (Kuske et al., 2009).

In home care settings, the interventions were organised either in small groups (Done \& Thomas, 2001; Ripich et al., 1998; Ripich et al., 1999) or individually (Barnes, 2016; Kouri et al., 2011; Liddle et al., 2012). Such training generally involved discussions and role-plays based on information from video vignettes or manuals/booklets.

\section{Outcome measures}

All studies conducted outcome assessments at baseline and post-intervention time points. 14 studies also conducted follow-up assessments to measure the stability of possible changes. 
Many outcome measures were in the form of subjective self-administered questionnaires. Some studies used a researcher-designed observation checklist or rating scale to assess realtime interactions (Burgio et al., 2001; Burgio et al., 2002; Sprangers et al., 2015) or videorecorded interactions (Alnes et al., 2011; Dampney, 2015; Ripich et al., 1999). One study employed a video-based test to assess carers' awareness about communication strategies, in which participants were asked to respond to scenes of communication breakdown (Done \& Thomas, 2001). In addition, external, more objective measures, such as documented use of physical restraints and sedative drugs (Kuske et al., 2009; McCallion et al., 1999) were adopted as outcome measures for people with dementia.

Regarding carer outcomes, all studies under review, except for Magai et al. (2002), measured the intervention effects on one or more aspects of carer communication knowledge, skills, and attitudes. Some studies also added the intervention effects on carer quality of life as a whole (Barnes, 2016), carer positive psychological states, such as satisfaction, positive aspects of caregiving, perceived ease of care-giving, self-efficacy, readiness/preparedness to provide care (Barnes, 2016; Broughton et al, 2011; Conway \& Chenery, 2016; Kouri et al., 2011; Liddle et al., 2012; Sprangers et al., 2015; Wells et al., 2000), or carer negative psychophysiological states, such as stress, burden, burnout, burden, distress, depression, anxiety, and somatic symptoms (Done \& Thomas, 2001; Kuske et al. 2009, Liddle et al. 2012, Magai et al., 2002; Sprangers et al., 2015; Ripich et al., 1998; Wells et al., 2000).

With respect to outcomes for people with dementia, some studies measured the intervention effects on their quality of life or wellbeing (Dampney, 2015; Liddle et al., 2012), mental/physical/social functions (Barnes, 2016; McCallion et al., 1999; Wells, et al., 2000), and other neuropsychiatric symptoms, such as depression, agitation, disorientation, irritability, and withdrawal (Liddle et al., 2012; Magai et al., 2002; Sprangers et al., 2015; Wells et al., 2000). Nine studies did not measure care-receiver outcomes because they did not directly involve people with dementia as their target participants.

\section{Effects on carer communication}

\section{Post-intervention results}

With comparable quantitative data, six studies, including four RCTs (Conway \& Chenery, 2016; Done \& Thomas, 2001; Kouri et al., 2011; Kuske et al., 2009), one NRCT (Broughton et al., 2011), and one CBA (Ripich et al., 1998), were included in a meta-analysis to examine the training intervention effect on carer communication between baseline and postintervention (Table 3). The pooled SMD was 0.89 (95\% CI 0.56, 1.22; $<<0.001$; Figure 2), indicating a statistically significant post-intervention improvement in communication in the intervention groups as compared to the control groups. There was significant between-study heterogeneity $\left(\mathrm{Q}=17.4 ; \mathrm{p}=0.004 ; \mathrm{I}^{2}=71.3 \% ; \tau^{2}=0.121\right)$. We found that study design fully explained the heterogeneity $\left(\mathrm{R}^{2}=99.9 \%\right)$ whereby the effect of intervention was smaller in RCTs (SMD 0.64, 95\% CI 0.43, 0.84) than in NRCT/CBA (SMD 1.38, 95\% CI 1.00, 1.77) (Table 2).

Among the ten studies that were not suitable for meta-analysis, eight studies (five RCTs, two NRCTs, and one CBA) demonstrated significant effects on one or more aspects of carer communication at post-intervention. The RCTs found significant improvement in the intervention group in terms of reduced communication difficulties experienced by carers (Barnes, 2016), overall communication skills especially in decreased negative statements (Burgio et al., 2001), behaviour management skills (Burgio et al., 2002), knowledge of support strategies (Liddle et al., 2012), and overall interaction behaviours (Wells et al., 2000). One CBA (Ripich et al., 1999) found a significant increase in knowledge of communication 
in dementia care, and one NRCT (Alnes et al., 2011) reported improvement of the intervention group in relation to an increase in supporting elements and a reduction in inappropriate elements. On the other hand, two RCTs found non-significant effects of the intervention on carer communication (McCallion et al., 1999; Sprangers et al., 2015).

\section{Follow-up results}

Five studies were eligible for a meta-analysis (Broughton et al., 2011; Conway \& Chenery, 2016; Kouri et al., 2011; Kustke et al., 2009; Ripich et al., 1998) to examine the intervention effect on carer communication between baseline and follow up outcomes (Table 4). The pooled SMD was $0.86(95 \%$ CI $0.67,1.05 ; \mathrm{p}<0.001$; Figure 3$)$, showing that the significant improvement in carer communication remained stable until weeks or months after the interventions. There was no evidence of significant between-study heterogeneity $(\mathrm{Q}=0.7$; $\left.\mathrm{p}=0.947 ; \mathrm{I}^{2}=0 \% ; \tau^{2}=0\right)$.

Among seven studies with follow-up data that were not included in the meta-analysis, improved carer communication was reportedly sustained after 2-6 months in three RCTs (Burgio et al., 2001; Burgio et al., 2002; Wells et al., 2000). However, the post-intervention improvement was not sustained in three other studies (Dampney, 2015; Liddle et al., 2012; Ripich et al., 1998).

\section{Effects on carer psycho-physiological states}

\section{Post-intervention results}

Five studies were included in the meta-analysis (Barnes, 2016; Done \& Thomas, 2001; Kuske et al, 2009; Magai et al., 2002; Ripich et al., 1998) to examine the intervention effect on carer negative psycho-physiological states (stress, burden, burnout, distress, depression, and anxiety) based on the comparison of the change between baseline and post-intervention outcomes (Table 5). It was found that the interventions had no significant effect on carer negative psycho-physiological states, with the pooled SMD being 0.34 (95\% CI $-0.16,0.84 ; \mathrm{p}$ $=0.182$; Figure 4). There was significant between-study heterogeneity $(\mathrm{Q}=12.3 ; \mathrm{p}=0.015$; $\left.\mathrm{I}^{2}=67.5 \% ; \tau^{2}=0.198\right)$. Study design did not contribute to the heterogeneity between studies (Table 2) and no other sources of heterogeneity were found.

A consistent non-significant effect was also found in the remaining two studies that measured carer negative psycho-physiological states. Specifically, two RCTs found no significant improvement in carer distress (Sprangers et al., 2015), or stress (Wells, et al., 2000).

\section{Follow-up results}

All of the six studies that had follow-up data on carer negative psycho-physiological states were eligible for the meta-analysis (Conway \& Chenery, 2016; Kuske et al., 2009; Liddle et al., 2012; Magai et al., 2002; Ripich et al., 1998; Wells et al., 2000) (Table 6). The interventions were found to have a minor effect on the reduction of carer stress, burden, burnout, distress, depression, and anxiety with the pooled SMD being 0.30 (95\% CI 0.01, $0.59 ; \mathrm{p}=0.042 ;$ Figure 5). There was moderate statistical heterogeneity between studies $\left(\mathrm{Q}=12.8 ; \mathrm{p}=0.078 ; \mathrm{I}^{2}=60.8 \% ; \tau^{2}=0.042\right)$. We did not find any source of the heterogeneity (Table 3) although the effect of training program appeared to be stronger in RCTs than in Non-RCTs. 


\section{Effects on the neuropsychiatric symptoms of people with dementia}

\section{Post-intervention results}

No significant effect was found on the post-intervention outcomes of care-receiver neuropsychiatric symptoms. Specifically, only one of the reviewed studies (Wells et al., 2000) measured post-intervention outcomes, and the authors found no significant effect (SMD $0.12,95 \%$ CI $-0.50,0.74$ ) of the intervention on the neuropsychiatric symptoms, such as depression, agitation, disorientation, irritability, or withdrawal, of people with dementia.

\section{Follow-up results}

In contrast, significant effects on the care-receiver neuropsychiatric symptoms were found in five out of six studies with follow-up data.

Three studies (Kuske et al., 2009; Sprangers et al., 2015; Wells et al., 2000) had follow-up data eligible for a meta-analysis to assess the intervention effect on the neuropsychiatric symptoms of people with dementia between baseline and follow-up outcomes (Table 7). It was found that the interventions had a positive effect on neuropsychiatric symptoms of people with dementia, with the pooled SMD being 0.62 (95\% CI 0.34, 0.90; $p<0.001$; Figure 6). There was no evidence of significant heterogeneity between studies $(\mathrm{Q}=1.5$; $\left.\mathrm{p}=0.480 ; \mathrm{I}^{2}=0 \% ; \tau^{2}=0\right)$.

Three other RCTs with quantitative data on neuropsychiatric symptoms of people with dementia reported different findings. The study by Magai et al. (2002) found no significant effects of the intervention on follow-up outcomes. On the other hand, Liddle et al. (2012) reported significant reduction in the frequency of depressive behaviours at follow-up, and McCallion et al. (1999) reported group x time interaction effects in many sub-aspects of depression, agitation, and other neuropsychiatric symptoms.

\section{Discussion}

The findings from this systematic review and meta-analysis indicate that communication interventions with a focus on regular care interactions have a positive impact on both carer outcomes and care-receiver outcomes, albeit to different degrees.

The intervention effects were found to be strongest on carer communication skills, knowledge, and attitudes, which tended to be sustained beyond the intervention period. This finding is encouraging, since improved carer communication was the primary outcome of almost all of the reviewed interventions. Generally, post targeted dementia communication training, carers have shown significant improvement in knowledge about dementia care communication, and in verbal as well as non-verbal skills in regular care interactions with people living with dementia, especially in providing support, giving instructions, facilitating understanding, and addressing behavioural symptoms. This beneficial impact of training on carers' communication has been widely reported in the literature (e.g. Roque, Ortiz, Araujo Mda, \& Bertolucci, 2009; Williams, 2006). It might have a link to carers' positive psychological states as quantitatively evidenced in many studies under review, such as increased caregiving readiness (Barnes, 2016), self-efficacy (Kouri et al., 2011), or positive aspects of caregiving (Liddle et al., 2012). Additionally, as many carers expressed their enhanced confidence in communicating with people with dementia, higher respect and empathy for these individuals, and increased ease of caregiving (e.g. Broughton et al., 2011; Conway \& Chenery, 2016; Dampney, 2015; Done \& Thomas, 2001; Liddle et al., 2012). 
With respect to possible explanations for the positive impacts on carer communication, the design of these interventions might play a part. The interventions under review tended to share some important features, including demonstration and/or presentation of the targeted knowledge and skills through video-based lectures or vignettes, followed by opportunities for discussion and practice (e.g. games or role plays) in an interactive face-to-face environment. This is quite consistent with the observations in previous systematic reviews, with special respect to the feature of interaction-based training (e.g. Vasse et al., 2010). In addition, these outstanding features of the reviewed interventions are in alignment with the widely recommended focus on promoting collaboration or co-construction of knowledge (Thomas, Menon, Boruff, Rodriguez, \& Ahmed, 2014) and meeting the varied learning needs of trainees (Chan, 2010) in training programs across disciplines. Future research and training programs might well consider opportunities to integrate interactive, collaborative and multimodal features into what is emerging as an increasingly online and self-directed healthcare learning environment (Davis, 2015; Lewis, Cidon, Seto, Chen, \& Mahan, 2014).

Notably, while both being characterized by a combination of multiple delivery/instructional methods, the training approaches slightly differed between residential care and home care settings. Specifically, effective residential care interventions were found to incorporate constructive feedback and counselling on real interactions between the care staff and people with dementia, as well as follow-up reinforcement or consultations as part of their training programs. These features were likewise highlighted in another review (Eggenberger et al., 2013) as contributing to effective training; however, these two features were not present in the home care interventions under review. This has an implication for future efforts of communication training in home care settings, regarding the inclusion of approaches found to be effective in residential care. The inclusion of individualised counselling on real interactions and follow-up reinforcement or consultations may serve to mitigate some of the potential isolation and other stressors of home care contexts as highlighted in the literature (Quinn, Clare, Pearce, \& van Dijkhuizen, 2008; Shanley, Russell, Middleton, \& SimpsonYoung, 2011).

A review of carer qualitative feedback on the interventions also revealed a number of important factors. The participating carers across studies tended to rate the communication programs positively, and their perceived strengths of the interventions were related to content, such as clarity and comprehensibility, contextualised video-based demonstrations, practicality and relevance, useful/handy training materials (Broughton et al., 2011; Conway $\&$ Chenery, 2016) and training delivery, such as constructive feedback on real interactions, group discussions, and hands-on practice (Dampney-Jay, 2015; Liddle et al., 2012). These interesting points could provide grounding for identifying success factors to inform similar communication training programs for carers of people living with dementia.

Although the reviewed interventions did not show a significant impact on carer psychophysiological states in post-intervention assessments, our meta-analysis shows that the reduction in carer negative psycho-physiological states (e.g. stress, burden, burnout, distress, depression, and anxiety) became significant in the follow-up assessments. Similarly, we found evidence that the interventions, especially those in residential settings, contributed to reducing the neuropsychiatric symptoms (e.g. depression, agitation, disorientation, irritability, and withdrawal) of people with dementia in the follow-up periods. These findings support the literature that has suggested the positive impact of communication training on the well-being of both carers and care-receivers in dementia settings (e.g. Auer et al., 2007;

Haberstroh et al., 2009; Krause et al., 2009). Perhaps evidence of follow-up effects was found due to the fact that such constructs as psycho-physiological and/or neuropsychiatric outcomes are unlikely to emerge immediately after the intervention. Therefore, it is suggested that 
future communication interventions employ repeated time measures to assess similar multifaceted and complex constructs. In addition, future communication interventions should incorporate neuropsychiatric symptoms of people with dementia in their research design, especially those in home care settings, where this receiver outcome seems to be neglected.

While the results might be encouraging, they should be interpreted with caution. What became evident throughout this systematic review was the variation in the methodological quality of the reviewed studies. Some studies had a low quality score, as likewise observed by previous researchers (Vasse, et al. 2010), but this might be because of the poor reporting quality, rather than the poor research methods. For example, only three (Barnes, 2016; Done \& Thomas, 2001; Kuske et al., 2009) out of 12 articles of RCTs included adequate description of the randomization and concealment process. Because the magnitude of bias associated with inadequate sequence generation and allocation concealment is usually high (Higgins \& Green, 2008), this lack of information has made it harder to make judgements about their possible bias. In addition, while blinding of participants was not always possible (Higgins \& Green, 2008), especially for this type of intervention, blinding of outcome assessors was not performed in two studies and it was not clear whether this was done in six other studies. Furthermore, in many studies where the communication outcome measures were newly developed by the author researchers, the reliability and validity of those measures were not justified or reported. The small sample size in some studies could have affected the statistical power and validity of their results as well. The pooled estimates may be somewhat underpowered due to the limited number of studies included in each meta-analysis (Green \& Higgins, 2011). However, our sensitivity analyses suggested that the hypothetical 'missing' studies were unlikely to greatly affect our conclusion.

Our systematic review highlights a need for more robust research concerning communication interventions relevant to regular care interactions between carers and people with dementia. Importantly, more consistent design and approaches to communication skills training, as well as reliable and valid measures for carer communication outcomes need to be developed for use in future research. This has also been emphasised in earlier reviews (Machiels, et al., 2017). Further, there is a need for greater clarity, transparency, and a more comprehensive approach to reporting research findings. It is therefore strongly suggested that future research encompass approaches such as the Consolidated Standards of Reporting Trials (CONSORT) statement (Turner et al., 2012) for parallel group randomised trials, to aid the reporting, critical appraisal and interpretation of research findings.

\section{Conclusion}

From this systematic review and meta-analysis, we have found strong evidence for the positive effects of communication training interventions on the communication skills and knowledge of carers in their regular care interactions with people living with dementia. These positive communication outcomes are likely to be sustained beyond the intervention period. In addition, there is some evidence for a reduction in carers' negative psycho-physiological states and the neuropsychiatric symptoms of people with dementia in the follow-up periods. Therefore, more research is needed on the impact of communication interventions, especially those that measure various outcomes of both carers and care receivers. It is also important to establish best practices in training design for such interventions in the context of dementia care, and to develop validated outcome measures and consistent reporting approaches. 


\section{References}

Allan, K., \& Killick, J. (2014). Communication and relationships: An inclusive social world. In M. Downs \& B. Bowers (Eds.), Excellent in dementia care: Research into practice (2nd ed.). New York: McGraw-Hill Education.

Allen-Burge, R., Burgio, D. L., Bourgeois, M., \& Nunnikhoven, J. (2001). Increasing communication among nursing home residents. Journal of Clinical Geropsychology, 7(3), 213-230. https://doi.org/10.1023/A:1011343212424

Alnes, R. E., Kirkevold, M., \& Skovdahl, K. (2011). Marte Meo Counselling: A promising tool to support positive interactions between residents with dementia and nurses in nursing homes. Journal of Research in Nursing, 16(5), 415-433. doi: $10.1177 / 1744987111414848$

Auer, S., Donabauer, Y., Zehetner, F., \& Span, E. (2007). Effect of a complex caregiver support intervention: A program of the Morbus Alzheimer Society Austria [German]. Entlastung pflegender angehoriger: Ein programm der M. A. S Alzheimerhilfe. Zeitschrift fur Gerontopsychologie und-psychiatrie, 20(2-3), 169-174. doi: 10.1024/1011-6877.20.2.169

Barnes, C. (2016). A pilot study to evaluate the effectiveness of an individualised and cognitive behavioural communication intervention for informal carers of people with dementia. (10302481 Ph.D.). University of Portsmouth (United Kingdom), Ann Arbor. Retrieved from https://researchportal.port.ac.uk/portal/files/5488758/Colin_Barnes_PhD_thesis_A pril_2016.pdf

Beer, L. E., Hutchinson, S. R., \& Skala-Cordes, K. K. (2012). Communicating with patients who have advanced dementia: Training nurse aide students. Gerontol Geriatr Educ, 33(4), 402-420. doi: 10.1080/02701960.2012.702165

Begg, C. B., \& Mazumdar, M. (1994). Operating characteristics of a rank correlation test for publication bias. Biometrics, 50, 1088-1101.

Berwig, M., Dinand, C., \& Halek, M. (2016). Application of MarteMeo Counselling to people with behavior variant frontotemporal dementia and their caregivers (AMEO-FTD)study protocol for a feasibility study. Journal of Neurochemistry, 138, 426.

Broughton, M., Smith, E. R., Baker, R., Angwin, A. J., Pachana, N. A., Copland, D. A., ... Chenery, H. J. (2011). Evaluation of a caregiver education program to support memory and communication in dementia: A controlled pretest-posttest study with nursing home staff. Int J Nurs Stud, 48(11), 1436-1444. doi: 10.1016/j.ijnurstu.2011.05.007

Bryan, K., \& Maxim, J. (2006). Communication disability in the Dementias. London: Whurr.

Buck, R., \& VanLear, C. A. (2002). Verbal and nonverbal communication: Distinguishing symbolic, spontaneous, and pseudo - spontaneous nonverbal behavior. Journal of Communication, 52(3), 522-541. https://doi.org/10.1111/j.14602466.2002.tb02560.x 
Burgio, L., Allen-Burge, R., Roth, D., Bourgeois, K., Gerstle, J., Jackson, E., \& Bankester, L. (2001). Come talk with me: Improving communication between nursing assistants and nursing home residents during care routines Gerontologist, 41(4), 449-460. doi: http://dx.doi.org/10.1093/geront/41.4.449

Burgio, L. D., Steven, A., Burgio, K. L., Roth, D. L., Paul, P. \& Gerstle, J. (2002). Teaching and maintaining behavior management skills in the nursing home. The Gerontologist, 42(4), 487-496.

Chan, J. F. (2010). Designing and developing training programs. CA: John Wiley and Sons.

Conway, E. R., \& Chenery, H. J. (2016). Evaluating the MESSAGE communication strategies in dementia training for use with community-based aged care staff working with people with dementia: A controlled pretest-post-test study. J Clin Nurs, 25(7-8), 1145-1155. doi: 10.1111/jocn.13134

Dampney-Jay, G. (2015). Improving nonverbal communication between caregivers and people with advanced dementia: The effect of staff training in intensive interaction on quality of life. (10076673 D.Clin.Psy.). Canterbury Christ Church University (United Kingdom), Ann Arbor. Retrieved from http://create.canterbury.ac.uk/13454/1/gail_dampneyjay_MRP_2015.pdf

Davis, J. (2015). Education through self-directed learning. Australian Nursing \& Midwifery Journal, 23(1), 26-27.

Done, D. J., \& Thomas, J. A. (2001). Training in communication skills for informal carers of people suffering from dementia: A cluster randomized clinical trial comparing a therapist led workshop and a booklet. Int J Geriatr Psychiatry, 16(8), 816-821.

Downs, M., \& Bowers, B. (2014). Excellent in dementia care: Research into practice (2nd ed.). New York: McGraw-Hill Education.

Downs, M., \& Collins, C. (2015). Person-centred communication in dementia care. Nurs. Stand., 30(11), 37-41. doi: 10.7748/ns.30.11.37.s45

Duval, S., \& Tweedie, R. (2000). Trim and fill: A simple funnel-plot-based method of testing and adjusting for publication bias in meta-analysis. Biometrics, 56(2), 455-463.

Eggenberger, E., Heimerl, K., \& Bennett, M. I. (2013). Communication skills training in dementia care: a systematic review of effectiveness, training content, and didactic methods in different care settings. Int Psychogeriatr, 25(3), 345-358. doi: $10.1017 / \mathrm{s} 1041610212001664$

Egger, M., Smith, G. D., Schneider, M., \& Minder, C. (1997). Bias in meta-analysis detected by a simple, graphical test. $B M J, 315: 629$. doi:https://doi.org/10.1136/bmj.315.7109.629

Green, S., \& Higgins, J. (2011). Cochrane handbook for systematic reviews of interventions version 5.1.0: The Cochrane Collaboration.

Haberstroh, J., Neumeyer, K., Schmitz, B., \& Pantel, J. (2009). [Development and evaluation of a training program for nursing home professionals to improve communication in dementia care]. Z Gerontol Geriatr, 42(2), 108-116. doi:10.1007/s00391-008-0527-x 
Higgins, J., \& Green, S. (Eds.). (2008). Cochrane handbook for systematic reviews of interventions. West Sussex: John Wiley \& Sons Ltd.

Hubbars, G., Cook, A., Tester, S., \& Downs, M. (2002). Beyond words: Older people with dementia using and interpreting non-verbal behaviour. J Aging Stud, 16(2), 155-167.

Jones, S. E., \& LeBaron, C. D. (2002). Research on the relationship between verbal and nonverbal communication: Emerging integrations. Journal of Communication, 52(3), 499-521. https://doi.org/10.1111/j.1460-2466.2002.tb02559.x

Kouri, K.K., Ducharme, F. C., \& Giroux, F. (2011). A psycho-educational intervention focused on communication for caregivers of a family member in the early stage of Alzheimer's disease: Results of an experimental study. Dementia, 10(3), 435-453. doi: $10.1177 / 1471301211408124$

Krause, K., Haberstroh, J., Neumeyer, K., Franzmann, J., Schmitz, B., \& Pantel, J. (2009). Tandem: Communication trainings for caregivers of dementia patients. Alzheimer's and Dementia, 1, 242.

Kuske, B., Luck, T., Hanns, S., Matschinger, H., Angermeyer, M. C., Behrens, J., \& RiedelHeller, S. G. (2009). Training in dementia care: A cluster-randomized controlled trial of a training program for nursing home staff in Germany. Int Psychogeriatr, 21(2), 295-308. doi: 10.1017/s1041610208008387

Levy-Storms, L., Harris, L. M., \& Chen, X. (2016). A Video-Based Intervention on and Evaluation of Nursing Aides' Therapeutic Communication and Residents' Agitation During Mealtime in a Dementia Care Unit. J Nutr Gerontol Geriatr, 35(4), 267-281. doi:10.1080/21551197.2016.1238430

Lewis, K. O., Cidon, M. J., Seto, T. L., Chen, H., \& Mahan, J. D. (2014). Leveraging e-Learning in medical education. Current Problems In Pediatric And Adolescent Health Care, 44(6), 150. doi:10.1016/j.cppeds.2014.01.004

Liddle, J., Smith-Conway, E. R., Baker, R., Angwin, A. J., Gallois, C., Copland, D. A., . . . Chenery, H. J. (2012). Memory and communication support strategies in dementia: Effect of a training program for informal caregivers. Int Psychogeriatr, 24(12), 19271942. doi: $10.1017 / \mathrm{s} 1041610212001366$

Machiels, M., Metzelthin, S. F., Hamers, J. P., \& Zwakhalen, S. M. (2017). Interventions to improve communication between people with dementia and nursing staff during daily nursing care: A systematic review. Int J Nurs Stud, 66, 37-46. doi: 10.1016/j.ijnurstu.2016.11.017

Magai, C., Cohen, C. I., \& Gomberg, D. (2002). Impact of training dementia caregivers in sensitivity to nonverbal emotion signals. Int Psychogeriatr, 14(1), 25-38.

McCallion, P., Toseland, R. W., Lacey, D., \& Banks, S. (1999). Educating nursing assistants to communicate more effectively with nursing home residents with dementia. Gerontologist, 39(5), 546-558.

McGuire, D. P. (1995). Effectiveness of an educational program to reduce stress of caregivers of patients with Alzheimer's disease. (1361492 M.S.), D'Youville College, Ann Arbor. 
Prince, M., Comas-Herrera, A., Knapp, M., Guerchet, M., \& Karagiannidou, M. (2016). World Alzheimer Report 2016: Improving healthcare for people living with dementia (pp. 1131). London: Alzheimer's Disease International.

Quinn, C., Clare, L., Pearce, A., \& van Dijkhuizen, M. (2008). The experience of providing care in the early stages of dementia: An interpretative phenomenological analysis. Aging \& Mental Health, 12(6), 769-778.

Ripich, D. N., Ziol, E., Fritsch, T., \& Durand, E. J. (1999). Training Alzheimer's disease caregivers for successful communication. Clin Gerontol, 21(1), 37-56.

Ripich, D. N., Ziol, E., \& Lee, M. M. (1998). Longitudinal effects of communication training on caregivers of persons with Alzheimer's disease. Clin Gerontol, 19(2), 37-55. doi: 10.1300/J018v19n02_04

Roque, F. P., Ortiz, K. Z., Araujo Mda, S., \& Bertolucci, P. H. (2009). Communicative strategies training effectiveness to caregivers of patients with dementia. Pro Fono, 21(3), 225-230.

Shanley, C., Russell, C., Middleton, H., \& Simpson-Young, V. (2011). Living through end-stage dementia: The experiences and expressed needs of family carers. Dementia, 10(3), 325-340. doi:10.1177/1471301211407794

Sprangers, S., Dijkstra, K., \& Romijn-Luijten, A. (2015). Communication skills training in a nursing home: Effects of a brief intervention on residents and nursing aides. Clin Interv Aging, 10(311-319). doi: https://doi.org/10.2147/CIA.S73053

Thomas, A., Menon, A., Boruff, J., Rodriguez, A.M., \& Ahmed, S. (2014). Applications of social constructivist learning theories in knowledge translation for healthcare professionals: A scoping review. Implementation Science, 9(54). doi: 10.1186/17485908-9-54.

Turner, L., Shamseer, L., Altman, D. G., Weeks, L., Peters, J., Kober, T., . . Moher, D. (2012). Consolidated standards of reporting trials (CONSORT) and the completeness of reporting of randomised controlled trials (RCTs) published in medical journals [Art. No.: MR000030]. Cochrane Database of Systematic Reviews 2012, Issue 11. doi:10.1002/14651858.MR000030.pub2

Vasse, E., Vernooij-Dassen, M., Spijker, A., Rikkert, M. O., \& Koopmans, R. (2010). A systematic review of communication stratefies for people with dementia in residential and nursing homes. Int Psychogeriatr, 22(2), 189-200. doi: $10.1017 /$ S1041610209990615

Ward, R., Vass, A., Aggarwal, N., Garfield, C., \& Cybyk, B. (2008). A different story: exploring patterns of communication in residential dementia care. Ageing and Society, 28, 629-651. https://doi.org/10.1017/S0144686X07006927

Wells, D. L., Dawson, P., Sidani, S., Craig, D., \& Pringle, D. (2000). Effects of an abilitiesfocused program of morning care on residents who have dementia and on caregivers. Advancing Geriatrics Nursing Practice, 48, 442-449.

Williams, K. N. (2006). Improving outcomes of nursing home interactions. Research in Nursing and Health, 29, 121-133. doi: 10.1002/nur. 
